Convergences francophones 6.2 (2020): 1-19

http://mrujs.mtroyal.ca/index.php/cf/index

\title{
La mort au tournant : enterrements, tombeaux et escales fatales dans l'inventaire du récit de voyage au XVIIIe siècle
}

\author{
Antoine Eche \\ Mount Royal University/ICD Tours
}

Dans le troisième tome des Voyages du Père Labat en Espagne et en Italie, le prêtre dominicain Jean-Baptiste Labat écrit : " Il faut à présent achever de décrire un enterrement tout entier » (III, 384). ${ }^{1}$ La nonchalance avec laquelle le père Labat livre cette phrase tout en soulignant la nécessité de la description à venir attire l'attention. En effet, cette phrase renvoie à la nécessité de satisfaire un objectif s'inscrivant visiblement dans l'horizon d'attente de son récit mais témoigne aussi d'un rapport à la mort qui n'est plus celui que nous connaissons dans le monde occidental aujourd'hui. Dans ses travaux sur les attitudes occidentales devant la mort, l'historien Philippe Ariès a ainsi montré quatre phases se succédant dans la diachronie. A la résignation devant une mort inévitable, familière et inscrite dans le destin de l'humanité, succèdent une vision plus personnelle de la mort et la prise de conscience de la mort de l'autre après celle de soi. Pour l'historien, l'émergence du regret et du souvenir de l'autre au XVIIIe siècle sont à la source du culte des tombeaux et des cimetières qui fleurira ensuite au XIXe siècle. Quant à la période contemporaine, elle se caractérise selon lui par un rejet de la mort, une mise à distance. Les voyageurs du XVIIIe siècle se situent donc à un moment charnière dans l'appréciation et la représentation de la mort. Et le récit de voyage à cette époque semble constituer un des lieux où l'on peut observer une des étapes du changement qui s'est opéré dans la perception occidentale de la mort. Mais pas seulement. Ainsi que nous l'évoquerons plus loin, ce genre participe activement à l'émergence de la nouvelle attitude face à la mort à la fin du XVIIIe siècle.

La particularité de notre approche est de partir du récit de voyage, ce « genre sans lois » (Le Huenen 11), ce « carrefour, parcours des savoirs sur le monde et sur soi » (Pasquali 131), qui semble avoir été malheureusement oublié par Philippe Ariès dans ses différents travaux sur le sujet, et ce en dépit du caractère récurrent

\footnotetext{
${ }^{1}$ Jean-Baptiste Labat (1663-1738) est une figure bien connue dans la littérature de voyage du XVIIIe siècle. Après avoir été ordonné prêtre et avoir enseigné les mathématiques et la philosophie à Nancy, il a effectué un long séjour aux Antilles en tant que volontaire (1693-1705) avant d'être rappelé à Rome pour y recruter de nouveaux missionnaires. Il passe rapidement en Espagne lors de son retour, resta quelques mois en Italie, revint en France, puis retourna en Italie pour plusieurs années avant de rentrer définitivement en France. Le père Labat profita de son séjour en Italie pour composer le récit de son voyage aux Antilles, Nouveau Voyage aux isles Françoises de l'Amérique (1722), qui connut un grand succès en France et il se spécialisa dans les récits de voyages, réels ou compilés. Les Voyages du Père Labat en Espagne et en Italie, publiés en 1730, paraissent à la suite de ce que l'auteur présente être une requête du marquis de $\mathrm{D}^{* * *}$, dont le nom demeure inconnu. On peut se demander s'il s'agissait d'une véritable demande ou bien d'un tour rhétorique en vogue dans les préfaces de ce type de récit pour en assurer l'intérêt par une tierce personne. Sur les 8 volumes des voyages, le premier est consacré à l'Espagne où il séjourna un peu plus de quatre mois, le second à un premier séjour de quelques mois en Italie et les 6 derniers relatent un séjour de 7 ans, toujours en Italie (I, vi-viii).
} 
de la thématique de la mort dans ce genre. ${ }^{2}$ Il ne s'agit pas ici de revenir sur la vieille métaphore associant la vie à un voyage et où la mort constituerait une étape pour les uns, le terminus pour les autres. C'est à la place de la mort dans l'une des composantes du récit de voyage, à savoir l'inventaire, que nous nous intéressons. Aux côtés de l'aventure et du commentaire, l'inventaire du voyage regroupe plusieurs thématiques censées saisir l'Autre du voyage dans son identité perçue. ${ }^{3}$ La majeure partie de ces thématiques existe depuis le début du genre, comme on peut le voir dans l'ouvrage d'Hérodote, L'Enquête, écrit au Ve siècle avant notre ère. Ces thématiques deviendront ensuite prescriptives au XVIe siècle dans des traités exposant les bienfaits du voyage d'éducation ainsi que des préceptes à suivre pour voyager efficacement (Stagl). Ces traités contiennent, de façon plus ou moins nette, des listes de choses à faire et à voir en voyage. Et c'est peut-être avec la liste que propose le chancelier anglais Francis Bacon dans son essai sur le voyage que l'on voit apparaître une liste de thématiques faisant la somme de pratiques antérieures. ${ }^{4}$ Cette liste est assez longue mais des regroupements sont possibles et révèlent des catégories thématiques telles que la politique, la justice, la religion, l'histoire, les arts et la culture, la technologie et le commerce :

Il est singulier que dans les traversées, où il n'y a rien à voir que le ciel et la mer, les gens tiennent leur journal, et qu'en voyageant sur la terre, où il $\mathrm{y}$ a tant de choses à observer, on néglige communément de le faire, comme s'il y avait lieu de noter des hasards plutôt que des réflexions. Qu'on prenne donc l'habitude de tenir son journal. - Les choses à voir et à étudier sont les suivantes : les cours des princes, notamment quand ils donnent audience aux ambassadeurs ; les cours de justice dans le temps qu'elles siègent et entendent plaider ; et de même les assemblées ecclésiastiques ; les églises et les monastères, et les souvenirs du passé qui s'y trouvent ; les remparts et fortifications des villes et des cités ; et aussi les havres et les ports ; les antiquités et les ruines ; les bibliothèques ; les cours et discussions des universités, s'il y en a ; les navires de commerce et de guerre ; les châteaux et les jardins d'apparat et de plaisance près des grandes villes ; les dépôts d'armes, les arsenaux, les magasins militaires ; les bourses et les marchés et

\footnotetext{
${ }^{2}$ Il y a 40 ans, François Hartog notait dans son étude sur l'Enquête d'Hérodote que « les rites funéraires ont toujours été un morceau de choix pour les observateurs, les voyageurs et les ethnologues» (Le Miroir d'Hérodote [1980], Paris, Gallimard, coll. Folio, 2001, p.228). Il s'appuyait sur un chapitre de l'ouvrage d'Emile Durkheim, Les Formes élémentaires de la vie religieuse (1912), pour ainsi relier le Ve siècle avant notre ère au XXe siècle. Par ce raccourci, il simplifiait la question de la représentation de la mort dans le genre du récit de voyage sans tenir compte de ses inflexions et, nous le verrons, de sa particularité au XVIIIe siècle.

${ }^{3}$ Sur ces trois notions, voir Ouellet (27).

${ }^{4} \mathrm{Ce}$ texte anglais datant de 1625 peut sembler éloigné de la période qui nous occupe ici mais en fait l'impact de Bacon sur la pensée des Lumières est bien connu. Rappelons que l'Encyclopédie de Diderot et d'Alembert reprend, avec quelques aménagements, le système de la division des sciences développé par Bacon et qui apparaît sous la forme de l'arbre des connaissances, le "Système figuré des Connaissances humaines". De plus, et de manière encore plus significative en ce qui concerne la conception du voyage au XVIIIe siècle, l'article "voyage" rédigé par le chevalier de Jaucourt reprend également l'incipit de l'essai de Bacon sur le voyage.
} 
les entrepôts ; les exercices d'équitation et d'escrime, l'instruction des soldats, etc ; les théâtres, ceux du moins que fréquente la bonne société ; les trésors de joyaux et de robes de cérémonie ; les cabinets de curiosités, bref tout ce qui est digne de mémoire dans les endroits qu'on visite ... Pour les cortèges, masques, banquets, mariages, obsèques, exécutions capitales, il n'est pas besoin de les mentionner, mais ils ne doivent pas être négligés. (93)

L'évocation de la mort et des rituels funéraires n'apparaît pas obligatoire mais reste cependant digne d'être mentionnée dans cette liste à vocation prescriptive. L'enquête sur l'Autre que constitue le récit de voyage ne peut se passer de consigner un vaste ensemble de pratiques socio-culturelles. Cette visée totalisatrice de la connaissance se retrouvera tout au long des siècles suivants dans différents traités. La représentation de la mort dans le récit de voyage aux côtés d'autres préoccupations n'est évidemment pas une nouveauté. ${ }^{5}$ Hérodote, déjà, décrit abondamment les funérailles des Scythes (IV, section 71-73) car, comme le note François Hartog, «La mort est signe d'altérité et elle intervient, dans le grand partage, toujours recommencé, entre le même et l'autre : elle est opérateur de différence ; soit: "Dis-moi comment tu meurs, et je te dirai qui tu es" » (229). C'est entre autre pour cette raison que le récit de Marco Polo au XIVe siècle, et ceux de Hans Staden et Jean de Léry au XVIe siècle incluent dans l'inventaire des scènes de funérailles, d'exécutions et d'anthropophagie qui se voient de plus illustrées, soit par le choix du copiste dans le cas du récit de Marco Polo, soit de l'auteur dans le cas des deux autres. ${ }^{6}$ Certains rites funéraires propres à certaines régions du monde deviennent également des topoï du genre, comme c'est le cas dans la représentation de la sati chez les voyageurs se rendant en Inde. ${ }^{7}$ La représentation de la mort est donc une thématique tant textuelle qu'iconographique dans le récit de voyage à l'aube du XVIIIe siècle.

\footnotetext{
${ }^{5}$ Christian Grosse, suivant Van Gennep, met en évidence le goût des antiquaires du XVIe siècle pour les rites de passage dont les funérailles et la constitution d'une littérature spécialisée et de là explique que «si les sociétés de l'Antiquité constituent bien dans ce traité comme dans les précédents le centre de gravité, les récits de voyage, cosmographies et témoins des conquêtes américaines du xvie siècle sont aussi largement sollicités et permettent d'étendre considérablement l'horizon de la comparaison ». Il poursuit en observant que «... la connaissance des usages funéraires des peuples nouvellement découverts par les Européens faisait des progrès. Si l'on ne repère pas à ma connaissance de traités spécifiquement consacrés à ces usages dans tel ou tel peuple, une rubrique les concernant figure presque toujours dans les cosmographies, les récits de voyages et le riche ensemble de textes décrivant les «mœurs et coutumes" des peuples du monde » (n.p.). Mais c'est là faire commencer l'histoire du récit de voyage bien tardivement. Si cette rubrique (ou thématique selon notre approche) est bien visible aux XVIe et XVIIe siècles dans les récits de voyages, c'est que la production de ces derniers a augmenté en fonction de l'augmentation des voyages extra-européens (mais aussi continentaux) et par-là, la masse d'informations ethnographiques.

${ }^{6}$ Voir entre autres Jean-Paul Duviols, « Premiers regards sur les sauvages (XVI ${ }^{\mathrm{e}}$ siècle) », América, vol. 50-1, p.13-25. https://doi.org/10.4000/america. 1789

${ }^{7}$ Voir Devika Vijayan, «Les anecdotes du suttisme - image d'une altérité féminine ambiguë », Convergences francophones, vol. 3., no.1, p.10-21.
} 
On comprend dès lors mieux la désinvolture exprimée par Jean-Baptiste Labat dans sa relation lorsqu'il introduit ce morceau attendu que constitue la description d'un enterrement à Tivoli. Et il n'y a rien d'étonnant ou de choquant à cela dans la mesure où la société occidentale jusqu'au XVIIIe siècle entretient une certaine familiarité avec la mort, que vient redoubler en quelque sorte le questionnement ethnographique des voyageurs. Si les épidémies, suicides, meurtres, exécutions publiques et accidents mortels faisaient partie du quotidien des voyageurs chez eux et n'étaient pas nécessairement vus comme étant exceptionnels, ils devenaient objets d'intérêt chez l'Autre et par-là producteurs d'un discours comparatiste, même implicite. Et c'est là qu'il faut poser une distinction et préciser de quelle mort on parle en reprenant la distinction faite par un autre historien, Michel Vovelle, entre la mort subie, la mort vécue et le discours sur la mort (1974). Seules les deux dernières notions importent ici. La mort vécue renvoie à « la pratique quotidienne [et aux] attitudes et comportements, dominés ou inconscients » tandis que le discours sur la mort est celui « des Églises, de ceux qui les contestent [et] sur un autre registre, de l'expression littéraire" » (Les attitudes 20). Par sa mise en texte, et donc son inscription dans une perspective singulière, la description de la mort vécue de l'Autre devient donc un discours sur la mort.

Les enterrements et les visites de tombeau reviennent assez régulièrement sous la plume des voyageurs. L'étude de ces deux objets de l'inventaire met en évidence deux discours sur la mort : celui visant directement l'Autre du voyage et celui exprimant le rapport du voyageur face à la mort de manière plus générale. A cela, il faut ajouter un troisième type de discours, plus rare, visant le voyageur luimême. Le premier type de discours est à l'œuvre dans le récit de Jean-Baptiste Labat. Et c'est celui curieusement de l'humour, voire de la moquerie. Ayant assisté à plusieurs cérémonies funéraires dans l'église des Jacobins à Tivoli, Labat se fait fort d'être expert en la matière (III, 384). Il décrit d'abord simplement les préparatifs du corps et les arrangements vestimentaires. De manière inattendue, il évoque ensuite la joie des curés de la ville : « Le curé de notre église ... et tous les curés de la ville se louaient beaucoup de cette année 1709. Il y avait longtemps qu'ils n'avaient fait une si copieuse moisson de morts » (III, 387). La raison est toute pratique : curés et religieux reçoivent des cierges, un peu d'argent mais en fait selon Labat, tout cela est bien peu (III, 388), ce qui finit par montrer la pauvreté des gens d'église en Italie mais aussi le peu de considération qu'ils accordent aux morts dans la mesure où le service offert correspond au montant des droits curiaux (coût des sacrements et des messes). Comme ceux-ci sont peu élevés, les curés « disent tout bas et en abrégé les prières » (III, 388). Le père Labat raconte ensuite une anecdote décrivant la mise en caveau d'une femme pour soulever en fait un problème d'ordre pratique lié au principe même de la mise en caveau des morts. En effet, du XIIIe au XVIIIe siècle, les riches sont enterrés dans l'église, dont on doit ouvrir le dallage pour accéder aux caveaux ; les moins riches sont enterrés autour de l'église, dans les aîtres, qui allaient donner naissance au cimetière ; et les pauvres finissent à la fosse commune (Ariès 52-65). Les caveaux des églises doivent donc être régulièrement vidés (Labat, III, 389) pour permettre à de nouveaux morts d'y 
séjourner quelque temps. Et la mise au caveau de cette femme va s'avérer problématique :

Ma curiosité me porta à m'approcher du caveau lorsqu'on en fit l'ouverture. A quelque odeur infecte près qui s'en exhala d'une manière assez supportable lorsqu'on leva la pierre, ma curiosité ne me reprocha rien. Je vis un très gros corps d'homme qui remplissait exactement l'ouverture, et qui touchait assurément la pierre quand elle était en place. Le Becamorto [fossoyeur] et ses compagnons furent assez longtemps à disputer s'ils refermeraient ce caveau et s'ils en ouvriraient un autre. Les avis étaients partagés, quoiqu'ils convinssent tous que les autres étaients également pleins, et que c'était à ce gros corps de faire place, puisqu'ils était le doyen. Ils se mirent donc en devoir de l'y contraindre, en le pressant fortement avec de gros bâtons; il obéit avec peine,et nous reconnûmes à une mauvaise odeur qu'il nous envoya qu'ils n'était pas content de changer de place. On mit promptement les pieds de la femme dans l'espace qu'il laissa vide, et deux forts Becamorti lui appuyant les bouts de leurs bâtons sur les épaules, la poussèrent avec tant de violence qu'ils lui firent trouver place malgré la résistance que les autres faisaient. Il y a apparence qu'elle creva en chemin faisant quelques-uns de ces corps qui nous envoyèrent un parfum qui aurait empesté le diable et tout l'enfer. Pour le coup, il fallut quitter la place, les Becamorti s'enfuirent hors de l'église, disant qu'il fallait laisser passer cette exhalaison avant de fermer le caveau. Je fus content au-delà de ce qu'on peut imaginer de ce que j'avais vu et senti. (III, 390-391)

Il est évident que cette anecdote vient dynamiser une description somme toute assez factuelle des enterrements. Le caractère quasi théâtral de la scène, avec le voyageur dans le rôle du spectateur, autorise le déploiement de divers registres comiques sur le thème de la décomposition des corps et la technique des fossoyeurs italiens. Mais plus que cela, cette évocation souligne à quel point les morts faisaient partie du monde des vivants, ne serait-ce que par leur odeur qui ne pouvait que se répandre dans les églises aux dalles mal scellées. Les ossements étaient parfois bien visibles à l'extérieur lorsque la terre était remuée pour accueillir de nouveaux occupants. Cette proximité autorise un humour qui serait jugé incongru de nos jours. L'ironie du commentaire final souligne aussi avec humour les risques auxquels pouvaient s'exposer des voyageurs peut-être un peu trop curieux.

A cette évocation riante de la mort, il est nécessaire d'en opposer une autre, celle visant la mort vécue d'un Autre plus lointain, non Européen. Ainsi, dans l'Histoire générale des voyages, compilation à l'origine anglaise traduite par l'abbé Prévost de diverses relations de voyages couvrant l'Afrique, l'Asie et les Amériques, on trouve plusieurs descriptions de cérémonies funéraires, certaines étant illustrées. C'est le cas de la section consacrée aux cérémonies mortuaires en Guinée. La cérémonie mortuaire royale est la seule à compter des sacrifices humains et la seule à se voir illustrée dans cette compilation; c'est dire qu'elle bénéficie d'une importance singulière (Fig.1). 


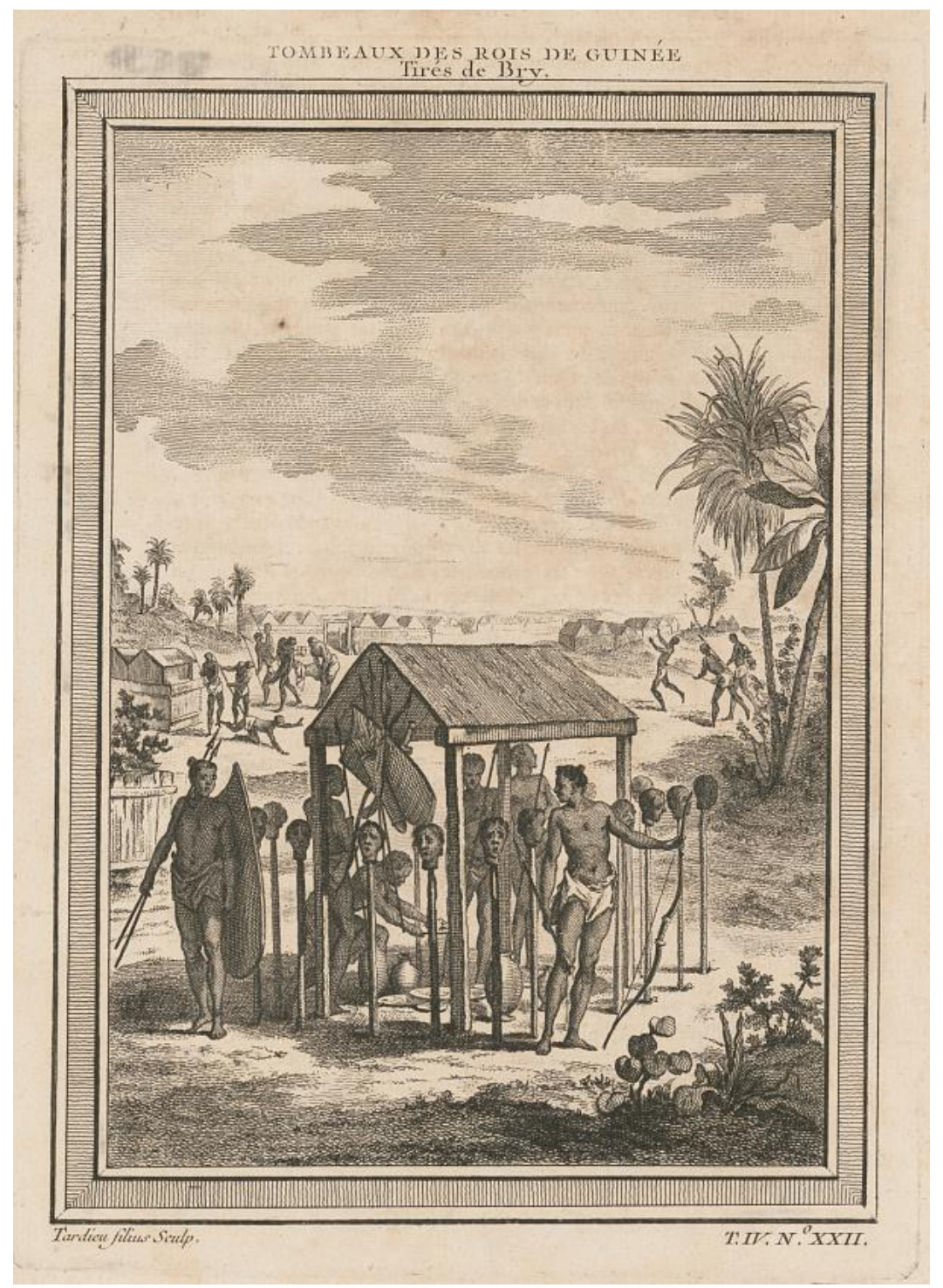

Fig.1 Tombeaux des rois de Guinée. Histoire générale des voyages. Paris, Didot, 1747, t. IV, p.152.

Library of Congress https://www.loc.gov/pictures/item/2003689332/

L'illustration fait partie des 65 images produites par Nicolas Cochin fils pour cette compilation. Elle n'est toutefois pas originale car la majorité des illustrations réalisées par Cochin était en fait des reprises d'illustrations souvent plus anciennes qui se trouvaient déjà dans la compilation anglaise qui reprenait, sans les modifier, des images déjà existantes. Celle-ci provient ainsi de la collection dite des Petits voyages de Théodore de Bry, qui constitue déjà un assemblage de diverses relations. ${ }^{8}$ L'illustration en question ne fait cependant pas partie des 18 planches de

\footnotetext{
8 "Regiae in Guinea Sepulturae Repraesentatio" dans Indiae Orientalis VI. Verat et Historicam Descriptionem Auriferi Regni Guinea, Francfort, 1604, n.p. L'auteur de cette relation, d'abord écrite
} 
l'original néerlandais (Iselin) et est aussi absente de la version française de 1605. C'est donc vraisemblablement de Bry qui a fait ajouter cette planche (Fig 2.).

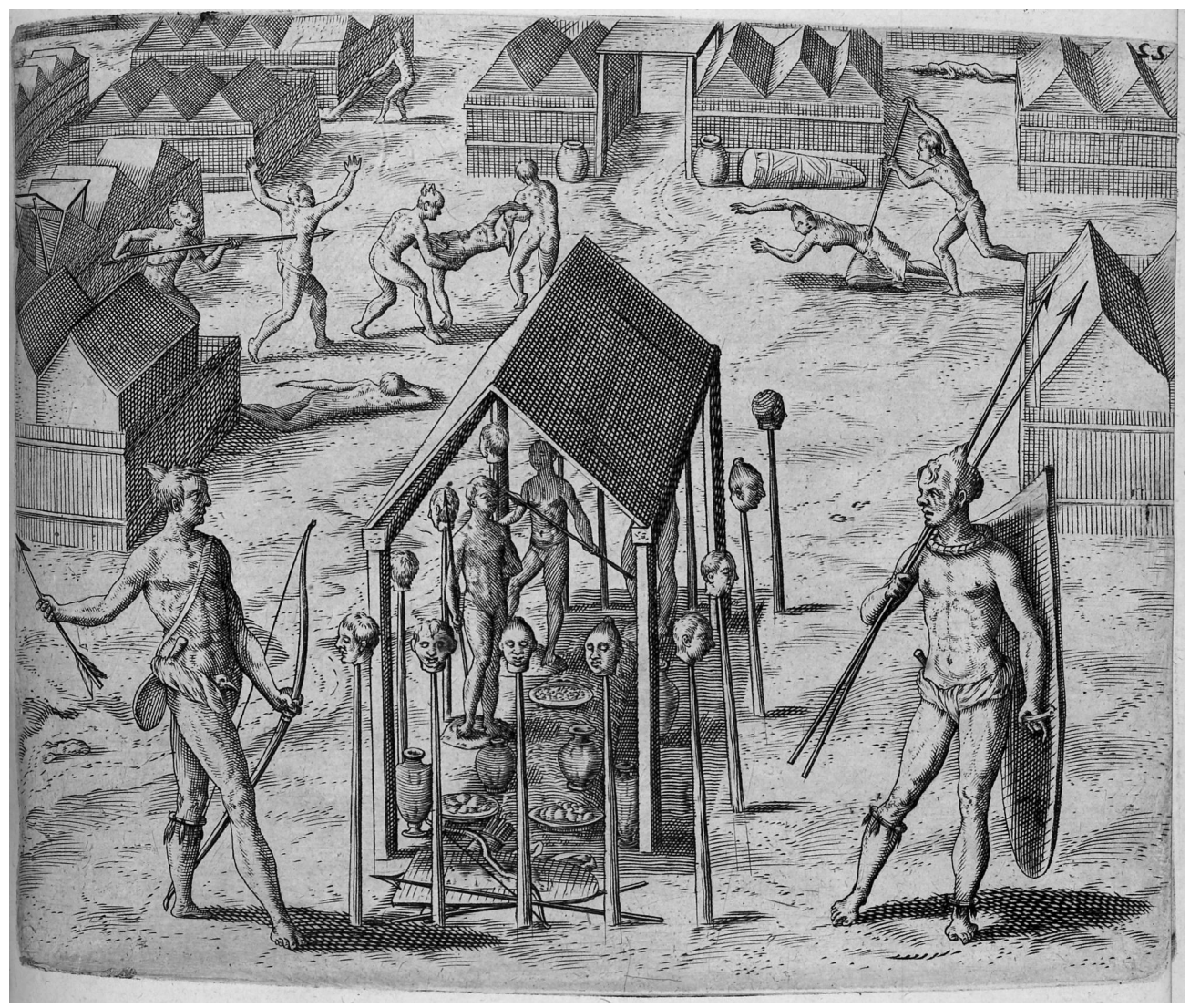

Fig 2. "Regiaein Guinea sepulturae repraesentatio", Slavery Images: A Visual Record of the African Slave Trade and Slave Life in the Early African Diaspora, $\mathrm{http} / / /$ slaveryimages.org/s/slaveryimages/item/2521

Sans pour autant apporter de profondes modifications au contenu de l'illustration, Cochin développe une esthétique plus en phase avec la composition des figures au XVIIIe siècle et le trait est beaucoup plus précis. Les traits des visages et des corps sont plus nets, et le paysage se voit développé selon la perception européenne d'un environnement naturel africain. Cochin modifie la perspective, annulant l'effet de plongée à l'œuvre dans l'image originale, créatrice d'une certaine distance, pour placer le lecteur (ou spectateur) au même niveau que les personnages représentés : l'effet de participation se voit accentué, tout comme le morbide de la scène (Eche Représentations 183-199). De même, la description textuelle de la cérémonie, sur

et publiée en néerlandais, est Pieter de Marees. Le traducteur de la version latine, Artus de Dantzig, sera pris pour l'auteur par le compilateur anglais de la version traduite par Prévost. Le texte français attribue donc ce texte à Artus, comme beaucoup à sa suite. L'ensemble de la collection des voyages de Theodore de Bry est depuis peu en ligne : https://bodmerlab.unige.ch/recits-etimages/debry/\#/petits-voyages/PVI 
laquelle repose l'illustration, n'a presque pas changé. Si une comparaison menée sur les premières versions française $(76-77,1605)$ et anglaise (II, 952, 1625) montre peu de variations, en revanche, la version latine de Theodore de Bry ajoute un élément concernant la disposition des têtes autour de la sépulture : celles-ci reposent sur des lances - "hastis" dans le texte (96) -, comme on le voit sur l'illustration. Dans les premières versions française et anglaise, les têtes sont disposées autour du roi, sans que ne soient donnés plus de détails. ${ }^{9}$ Le détail rajouté par de Bry sera repris dans l'original anglais que Prévost traduit ici quasiment littéralement. ${ }^{10} \mathrm{La}$ question des sacrifices humains modifie en fait le discours sur la mort vécue de l'Autre au point que la différence entre les Européens et les Africains ne se posent plus en termes de religion mais en termes moraux pour (ré)affirmer la supposée barbarie des Noirs (Law 53 ; Halpern). La conjonction entre l'image et le texte de la mort de l'Autre renforce ici un discours visant à réaffirmer la supposée infériorité des Noirs vis à vis des Blancs (Eche L'image 214-216). Et ici le principe de la compilation qui fait cohabiter plusieurs textes, et donc plusieurs témoignages réels, voire inventés, permet de construire une gradation dans l'horreur morale proportionnelle au degré de barbarie supposé des peuples guinéens dans leur ensemble qui permet au compilateur de conclure que " Cette barbare coutume s'observe avec plus ou de moins zèle, au long des Côtes de Guinée » (HGV, IV, 154). En traduisant assez fidèlement cette portion de l'ouvrage, Prévost valide in fine l'attitude du compilateur anglais et, du fait de la prégnance de la langue française en Europe à cette période, démultiplie son impact sur un lectorat plus vaste.

Le récit de voyage joue également un rôle actif dans le changement d'attitude face à la mort. Il nous faut ici quitter l'Afrique et revenir en Europe, en Italie d'abord puis en France, afin d'évoquer la visite des tombeaux des hommes de lettres. On voit dans un premier temps que la visite de tombeaux s'inscrit tout à fait dans l'inventaire du voyage. Ainsi, dans le récit du voyage en Italie publié par l'avocat Pierre Jean Grosley en 1764, on retrouve des descriptions de tombeaux de saints mais aussi de celui de Virgile, l'auteur de l'Enéide et des Géorgiques, près de Naples. Cette visite s'inscrit dans un courant dit antiquisant, hérité de la Renaissance, et participe à l'inventaire des traces de l'Antiquité sous toutes ses formes. Au XVIIIe siècle, cette mode reprend de l'essor avec la (re)découverte des sites d'Herculanum et de Pompéi (Grell). Selon Pierre Jean Grosley, "des anciens monuments de Naples, le plus entier et le plus vénérable est le tombeau de Virgile" (III, 1764, 78). La description est assez factuelle et vise essentiellement l'architecture du mausolée. Grosley note toutefois un "prodige" concernant ce

\footnotetext{
${ }^{9}$ En français : «Les têtes des occis mect on tout autour de la sepulture pour aornement ... » (76). En anglais: "The heads of the dead men and women, are set about the sepulchre and grave of the king, for a great honour and braverie unto him" (962).

10 "The heads of the persons killed are not buried with the bodies but fixed on poles round the grave, which they think a very honourable ornament" (II, p. 662); « On n'enterre point les têtes avec leur corps. On les plante autour du monument, sur des pieux, comme le plus honorable de tous les ornements funèbres » (IV, 153). Ainsi qu'il l'indique dans ses sources, le compilateur anglais s'appuie sur la version latine de Theodore de Bry.
} 
monument : c'est la présence d'un laurier venant couronner la coupole. Le mort semble ainsi honoré à jamais, ce qui conduit notre voyageur, quelque peu facétieux, à citer un vers d'une comédie d'Alexis Piron, intitulée La Métromanie (1738), dans laquelle le personnage principal a la manie de faire des vers dans n'importe quelle situation.

Cependant le laurier de Virgile toujours vigoureux, toujours renaissant, se perpétue, répare ses pertes journalières, Croit et s'élève encore au sommet du Parnasse. (III, 78)

La visite de ce tombeau semble être un passage obligé pour tout voyageur se rendant dans les environs. En effet, on en trouve une description accompagnée d'une illustration dans un ouvrage de la fin du XVIIe siècle et considéré comme l'un des best-sellers du voyage en Italie au XVIIIe siècle, à savoir le Nouveau voyage d'Italie de Maximilien Misson, que tout voyageur se devait d'avoir lu ou emporté avec soi. La description est moins étendue que chez Grosley mais les deux auteurs soulignent le doute qui plane sur le fait que ce mausolée soit bien celui de Virgile (Grosley, 80-82; Misson, 1691, I, 317-319). Encore réduite à un simple élément de l'inventaire, la visite au tombeau va changer de statut une dizaine d'années plus tard. En effet, un second type de visite de tombeau apparaît dans le dernier quart du siècle : il s'agit des voyageurs se rendant sur la tombe de JeanJacques Rousseau, située sur l'Île des Peupliers dans les jardins d'Ermenonville créés par Stanislas Girardin. Assimilé à un pèlerinage, le voyage au tombeau de Rousseau constitue une excursion à faire depuis Paris ou bien un passage obligé pour ceux circulant en Europe - comme le suggère la présence de visiteurs dans l'illustration ci-dessous —avant que son corps ne soit transféré au Panthéon en 1794 (Fig. 3). 


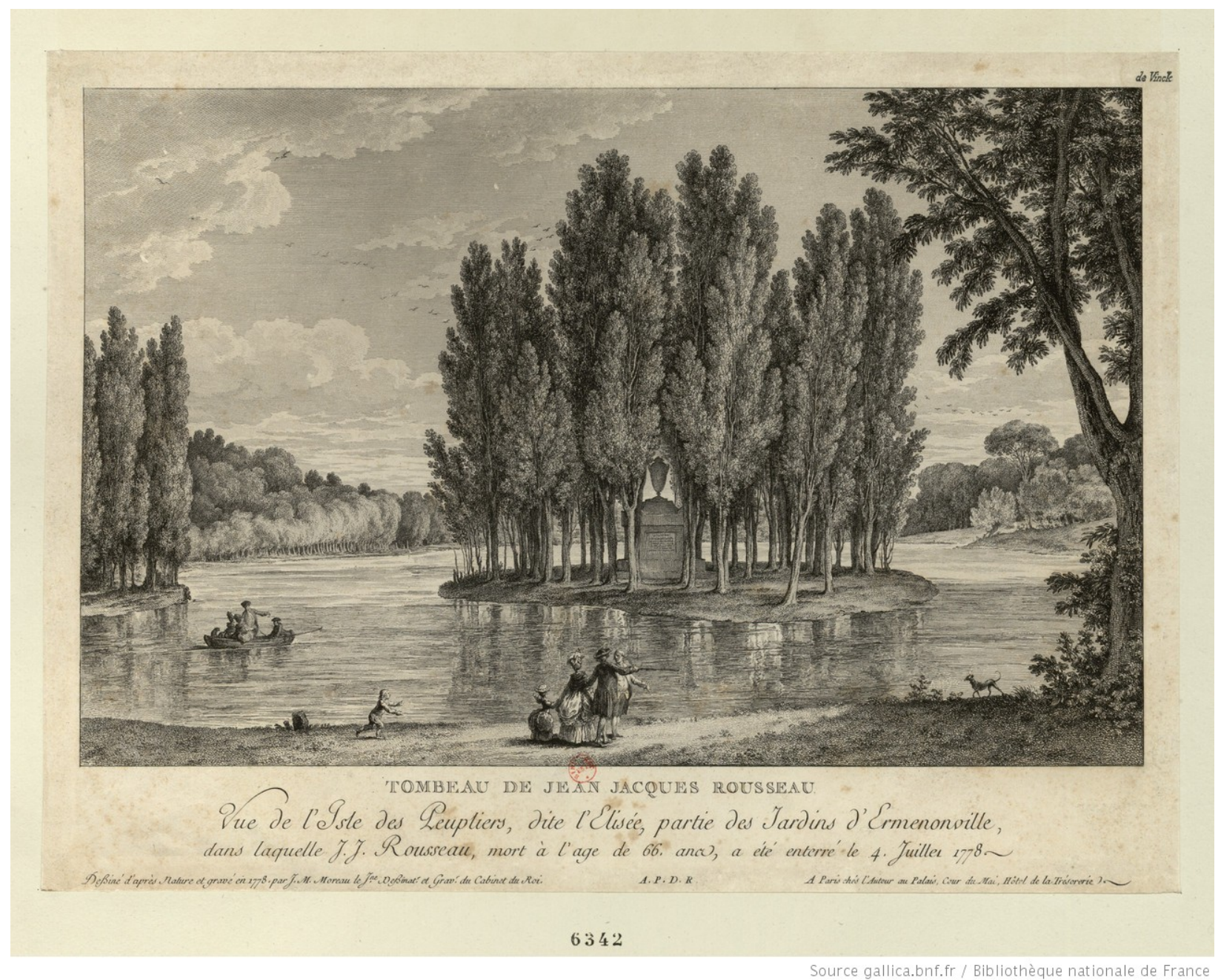

Fig. 3 Tombeau de Jean-Jacques Rousseau par Jean-Michel Moreau. https://gallica.bnf.fr/ark:/12148/btv1b6950591s

Ces visites ont déjà fait l'objet d'études ayant permis de dégager certains traits (Ridehalgh ; Trousson) mais sans toutefois prendre en compte leur inscription dans la dimension générique du récit de voyage. ${ }^{11}$ En effet, au lendemain de la mort du philosophe en 1778, la visite au tombeau devient l'objet même de certaines relations, supplantant ainsi les autres thématiques généralement évoquées dans le récit de voyage continental européen. Des titres tels que A Tour to Ermenonville (1785) ou Voyage à Ermenonville (1788) montrent bien à quel point la visite au tombeau de l'homme de lettres est devenue la raison d'être du récit. ${ }^{12}$ Dans l'évocation des jardins et du tombeau, les voyageurs manifestent une sensibilité aux

\footnotetext{
${ }^{11}$ Nous nous sommes interrogés sur cette question dans notre article en cours d'évaluation « La visite de trois Anglais au tombeau de Jean-Jacques Rousseau à Ermenonville : développement et saturation d'un imaginaire dans le voyage en France au XVIIIe siècle ».

${ }^{12}$ Cet ouvrage de Pierre Letourneur parait de façon posthume en 1788 dans le premier tome des œuvres complètes de Rousseau de l'édition Poinçot et sert de préface générale.
} 
accents rousseauistes qui se retrouve également chez d'autres voyageurs arpentant les lieux évoqués dans la Nouvelle Hélö̈se ou dans Les Rêveries du promeneur solitaire. Ce discours sur la mort de toi, pour reprendre la terminologie de Philippe Ariès, célèbre une nouvelle attitude face à la mort qui se développera au XIXe siècle avec le culte des tombeaux et des cimetières. S'il ne constitue pas un nouveau genre en soi, ce type de récit illustre, s'il le fallait encore, la grande souplesse du genre. C'est en fait une variation du genre axé sur une thématique principale, comme ce fut le cas pour d'autres thématiques telles que les arts ou la musique.

Le troisième type de discours est le plus rare puisqu'il implique la mort d'un voyageur ayant au préalable entrepris de relater son voyage (ou de le faire relater) sans qu'il ne puisse l'accomplir complètement. Le cas le plus célèbre au XVIIIe siècle est sans doute celui du capitaine James Cook, mort lors de son troisième voyage autour du monde à bord du Resolution dans la baie de Kealakekua le 14 février 1779. En modifiant la terminologie de Philippe Ariès, on pourrait parler ici de la mort de soi chez l'Autre. A la différence d'un Magellan, par exemple, mort en 1521 aux Philippines, la mort de Cook va connaître une publicité et une diffusion sans précédent de par les conditions de diffusion et de circulation de l'information de l'époque (presse imprimée, presse manuscrite, ouvrages, correspondance, etc.) ainsi que par la perception de l'apport du navigateur à l'épistémologie des Lumières. La nouvelle de sa mort va atteindre l'Angleterre et le reste de l'Europe le 10 janvier 1780 dans une lettre rédigée par le capitaine du Discovery, Charles Clerke, qui sera entre temps décédé de maladie. Cette lettre constitue pendant environ un an la seule relation des faits entourant la mort de Cook : suite au vol d'un canot, le capitaine se rend à terre pour en discuter avec le chef des insulaires et les échanges jusqu'alors cordiaux se détériorent au point que Cook utilise son arme à feu contre l'un des indigènes, provoquant alors une mêlée dans laquelle il trouva la mort. Pour des raisons liées à l'exécution des planches illustrées, le récit officiel ne paraîtra pas avant 1784 (Williams 16). La parution très rapide d'articles de journaux et de récits non officiels (16-22) va constituer un premier discours, de type épitextuel, qui témoigne entre autres de l'engouement et de la manne financière que représente la tragique fin d'un voyageur reconnu en Europe pour son apport à l'édifice du savoir des Lumières ${ }^{13}$. Parce qu'elle relève de l'événementiel du voyage, la mort du voyageur va se traduire sous le mode du récit circonstancié de ses derniers instants, tant d'un point de vue textuel que visuel. On trouve ainsi dès 1781 une illustration dépeignant les tous derniers instants de la mort de Cook dans le récit tiré du journal d'un lieutenant du Discovery, Journal of Captain's Cook Last Voyage de John Rickman ${ }^{14}$ (Fig. 4) Le capitaine est allongé sur le rivage

\footnotetext{
${ }^{13}$ Le livre de voyages constitue un véritable marché pour certains éditeurs tels, en France, CharlesJoseph Panckoucke, qui publie entre autres les traductions des voyages de Cook dans des éditions luxueuses et richement illustrées. Le sensationnel de la disparition du navigateur vient renforcer l'intérêt d'un lectorat déjà demandeur mais aussi piqué par la curiosité.

${ }^{14}$ La version française parait en 1782 sous le titre : Troisième voyage de Cook ou Journal d'une expédition faite dans la mer pacifique du sud et du nord en 1776, 1777, 1778, 1779 et 1780 (Paris, Pissot et Laporte). Nous citons plus loin la troisième édition, parue sous le même titre chez Poinçot et Belin en 1783.
} 
et est sur le point d'être battu à coup de massues tandis que ses hommes fuient devant la supériorité numérique des insulaires. La lecture du texte donne plus de détails, notamment sur les conditions de la mort du capitaine : celui est d'abord assommé puis poignardé dans le dos $(1781,319)$, ce que l'image ne montre pas, tout comme l'enlèvement de son corps par les insulaires. L'auteur poursuit en évoquant la fin de l'échauffourée et conclut la scène par la déploration du "plus illustre navigateur de l'Angleterre, et même d'aucune nation du monde" (1783, $362)^{15}$ et l'invitation au lecteur éclairé à verser quelques larmes sur le sort tragique du capitaine :

Lecteur, si tu sais apprécier ce grand homme; si tu es sensible à l'affliction de la nombreuse famille qui le pleure, tes yeux se mouilleront de larmes ici comme les miens; tu regretteras qu'après avoir échappé à tant de dangers sur la mer, après avoir affronté mille fois la mort, l'intrépide Cook périsse de la main d'un sauvage qui vient le poignarder par derrière. (1783, 362363)

Il faut noter ici que la version française modifie et adoucit singulièrement le texte anglais, dans le sens où l'auteur de ce dernier inscrit le chagrin dans une logique patriotique et développe explicitement une isotopie de la lâcheté supposée des insulaires, sous-entendue pour le lecteur européen qui voit dans une agression dans le dos le non-respect d'un code d'honneur tout européen :

Reader, if thou hast any feeling for thy country in the loss of so great, so illustrious a navigator, or any tenderness for those he has left to lament his fate, thou will drop with me a tear at this melancholy relation; especially when thou reflectest that he, who had braved dangers, and who had looked at death in a thousand forms, should at last be cut off by the hands of a cowardly savage, who dreading the impetuosity of his rage, came behind him, and ruffian-like, stabbed him in the back. $(1781,320)$

${ }^{15}$ Dans l'original : “... the greatest navigator that this or any other nation could boast ...” (1781, $320)$. 


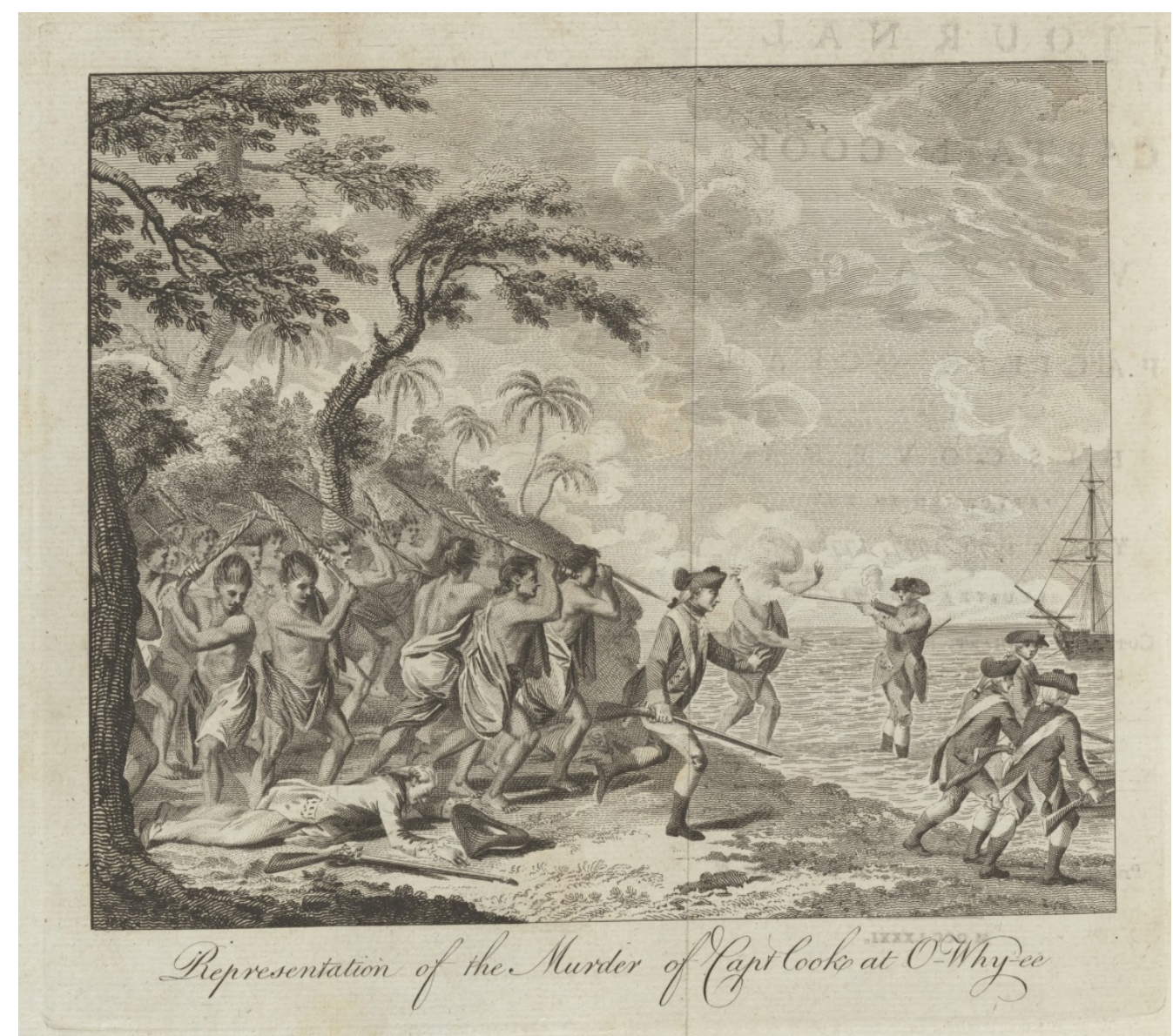

Fig. 4. Representation of the Muder of Captain Cook at O-Why-ee. Journal of Captain's Cook Last Voyage to the Pacific Ocean on Discovery. Londres, Newberry, 1781, (n.p). John Rickman, National Library of Australia, nla.obj-189475722

Entre 1780 et 1784, l'intérêt du public ne faiblit pas : la publication du récit officiel est un véritable succès malgré son prix élevé ; des éditions abrégées et moins onéreuses suivent très rapidement et la presse relaie les grands moments du voyage par épisode (Williams 22-24). Ces publications vont particulièrement cibler les événements précédant la mort de Cook ainsi que le récit de cette dernière. Le récit officiel est accompagné, selon l'habitude, de tout un appareil péritextuel. La fonction de ce dernier dépasse le cadre qui est généralement le sien en visant à l'héroïsation du capitaine anglais : tout d'abord dans l'introduction générale où Cook est dépeint comme le martyr de la connaissance ( $A$ Voyage I, ii-iii) et ensuite dans l'éloge proprement dit où il devient le modèle des voyageurs (I, lxxxii lxxxix). La traduction française du récit officiel, effectuée par Jean Nicolas Démeunier, résume assez bien l'état du discours sur la mort de Cook. A la différence de la version officielle anglaise, celle-ci prend pour frontispice l'illustration de la mort de Cook par John Webber, artiste engagé à bord lors du voyage (Fig.5). A la différence de l'image se trouvant dans le récit de Rickman, le capitaine se trouve dans une pose plus noble, debout, la main tendue vers ses hommes comme pour les empêcher de tirer sur les insulaires. La clarté du 
personnage le détache de la scène et en fait le point le focal, tout comme l'homme qui s'apprête à le poignarder. Le mythe est en marche. Et le texte, en anglais comme en français, confirme cette version illustrée :

Notre malheureux commandant se trouvait au bord de la mer la dernière fois qu'on l'aperçut d'une manière distincte; il criait aux canots de cesser leur fau et d'approcher du rivage afin d'embarquer notre petite troupe. S'il est vrai que les soldats de marine et les équipages des canots avaient tiré sans son ordre, et qu'il voulait prévenir une nouvelle effusion de sang, comme quelques-uns qui furent de l'action l'ont cru, il est probable qu'il fut la victime de son humanité : on observa en effet que tandis qu'il regardait les naturels en face, aucun d'eux ne se permit de violences contre lui, mais que s'étant retourné pour donner des ordres aux canots, il fut poignardé par derrière et tomba le visage dans la mer. (1785, III, 437-438)

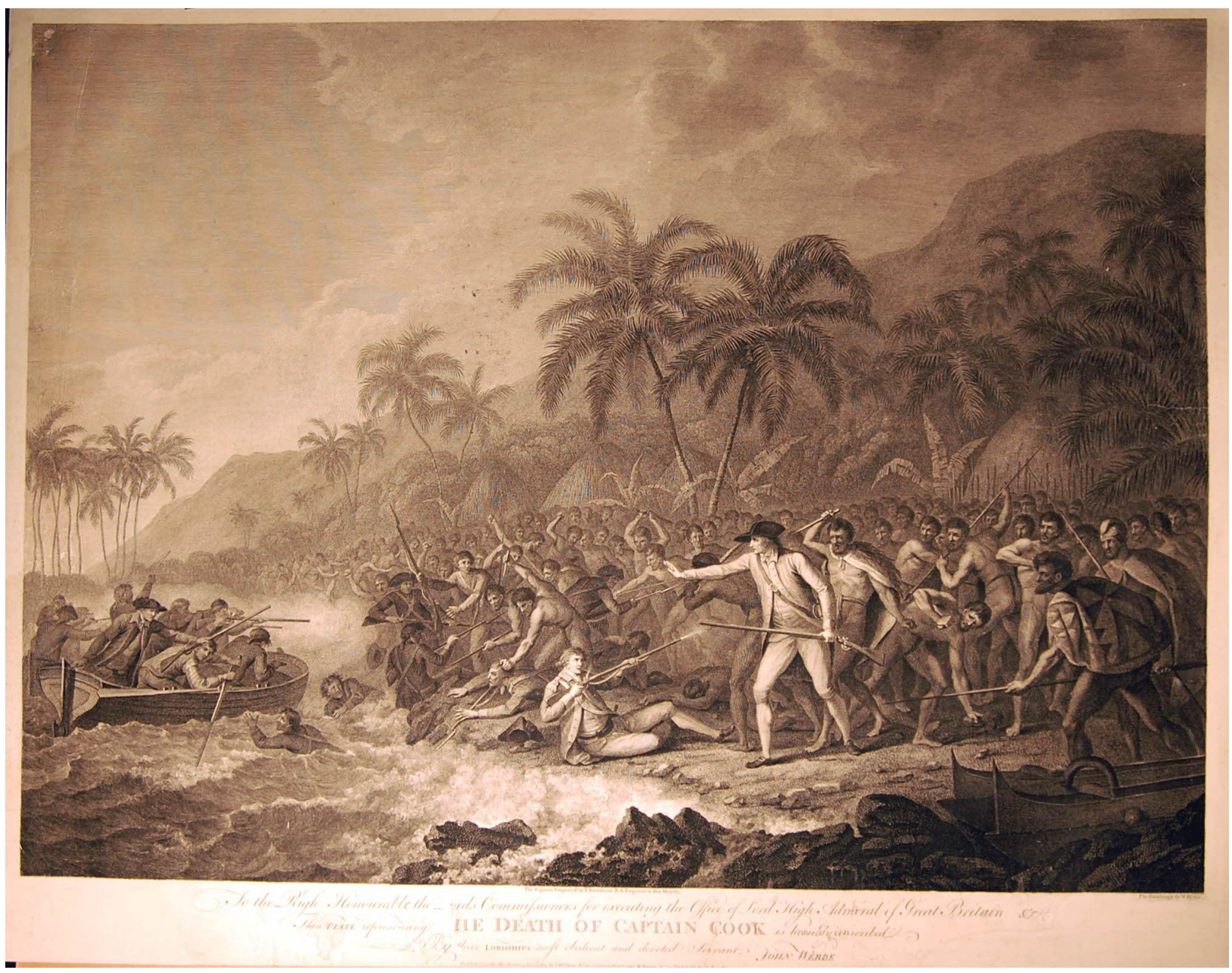

Fig.5. The Death of Captain Cook. John Webber, Londres, 1784.

https://research.britishmuseum.org/research/collection_online/collection_object_details.a spx?objectId=3189992\&partId=1 $@$ Trustees of the British Museum. 
Démeunier traduit ici fidèlement le récit anglais ${ }^{16}$ qui se poursuit par une biographie tout à la gloire du navigateur. Tout comme dans le cas du récit de John Rickman, le positionnement de l'illustration au début du péritexte de la version française bouscule l'ordre chronologique du récit et sa dynamique iconographique en imposant une lecture téléologique du voyage menant au récit textuel de la mort du navigateur. En plus de l'introduction, et comme pour remplacer la suppression de l'éloge, la version française est accompagnée d'une préface du traducteur qui s'ouvre par la mise en scène de la figure héroïque du capitaine : «La géographie du globe était couverte de ténèbres, lorsque l'immortel Cook a commencé ses voyages autour du monde. Ses deux premières expéditions nous ont fait connaître une multitude de côtes et d'îles nouvelles, et la troisième a encore peut-être été plus heureuse à cet égard » (Troisième voyage, I, i) Grâce au récit, le voyageur transcende la mort (il est immortel) mais c'est par la mort qu'il atteint ce statut. Comme l'observe l'historien Glyn Williams, “... only after his death was there widespread appreciation of Cook's achievements" (3). Et, même si les voyageurs se rendant à la baie de Kealakekua seront bien moins nombreux que ceux ayant arpenté les sentiers du parc d'Ermenonville pour se recueillir sur la tombe de Rousseau, le lieu de la mort de Cook, avec son inscription et son monument, fera l'objet d'un pèlerinage volontaire ou non (Williams 98-109), inscrivant ainsi la mort du navigateur dans l'inventaire du voyage. ${ }^{17} \mathrm{Il}$ est toutefois notable que pour certains voyageurs, comme La Pérouse - qui périt en 1788 sur les récifs de l'île de Vanikoro - la notion de pèlerinage ne sera pas consacrée par la visite du lieu où Cook perdit la vie mais par la route empruntée et le souvenir du navigateur anglais. Quittant l'île de Pâques en direction de Maui, il observe que "notre route était à peu près parallèle à celle du capitaine Cook en 1777 lorsqu'il fit voile des îles de la Société pour la côte du Nord-Ouest de l'Amérique" (II, 104) et, approchant de l'archipel hawaïen, déclare : “... tous les navigateurs doivent un tribut d'éloges à sa mémoire ; comment m'y refuser au moment d'aborder le groupe d'îles où il a fini si malheureusement sa carrière !" (II, 108). Le voyage devient alors un voyage dans la mémoire tragique du voyage et de sa bibliothèque, et la mort de l'alter ego du voyageur, une variante thématique dans l'inventaire.

De la nonchalance de Jean-Baptiste Labat dans sa description d'un enterrement en Italie à l'engouement suscité par la mort de Cook et à son héroïsation en passant par les récits focalisés sur la visite au tombeau d'un homme de lettres ayant atteint un statut sans précédent de son vivant, on observe ainsi comment le thème de la mort connaît un mouvement dynamique au sein du genre du récit de voyage en phase avec le développement des attitudes européennes devant la mort. Il n'est sans doute pas étonnant que le récit de voyage rejoigne lui-aussi la masse des discours sur la mort de manière plus évidente à la fin du XVIIIe siècle avec "le retour des

\footnotetext{
${ }^{16}$ Voir III, 45-46 pour l'original.

${ }^{17}$ Le missionnaire William Ellis observe en 1823 que "Few intelligent visitors leave Hawaii without making a pilgrimage to the spot where [Cook] fell" (IV, 137).
} 
idées noires" (Vovelle, 1992, 116) que connaît la période. En impactant les pratiques de la mobilité, ces attitudes s'affichent dans les récits qui en retour les perpétuent et les célèbrent. Dans ce cas-ci, le récit de voyage acquiert une fonction sociale qui dépasse sa mission informative ou, dans le cas du récit de pèlerinage, spirituelle : il devient un guide des attitudes devant la mort. 
Convergences francophones 6.2 (2020): 1-19

http://mrujs.mtroyal.ca/index.php/cf/index

\section{Bibliographie}

A Tour to Ermenonville; containing, besides an Account of the Palace, Gardens and Curiosities of Chantilly, And the Marquis de Girardin's beautiful Seat of Ermenonville, A particular Description of the Tomb of J.J. Rousseau, with anecdotes (never before published) of that celebrated and singular Man. London, T. Beckett, 1785.

Ariès, Philippe. L'homme devant la mort. Paris, Seuil, 1977.

Bacon, Francis. Essays. Collection bilingue des classiques étrangers, Paris, Aubier, 1948.

Cook, James. A Voyage to the Pacific Ocean. Undertaken, by the Command of His Majesty, for making Discoveries in the Northern Hemisphere. 3 volumes. London, W. and A. Straham, 1784.

---. Troisième voyage de Cook. 5 volumes. Traduit par Jean-Nicolas Démeunier, Paris, Hôtel de Thou, 1785.

Duviols, Jean-Paul. « Premiers regards sur les sauvages (XVI ${ }^{\mathrm{e}}$ siècle). » América, vol. 50-1, pp.13-25. https://doi.org/10.4000/america.1789

Eche, Antoine. « La visite de trois Anglais au tombeau de Jean-Jacques Rousseau à Ermenonville: développement et saturation d'un imaginaire dans le voyage en France au XVIIIe siècle » (article soumis pour publication).

---. Représentations de l'altérité dans l'Histoire générale des voyages de l'abbé Prévost. Paris, Le Manuscrit, 2017.

---. « L'image ethnographique africaine de l'Histoire générale des voyages. » SVEC, vol. 5, 2009, pp. 207-222.

Ellis, William. Polynesian researches, during a residence of nearly eight years in the Society and Sandwich Islands. vol. 4. Londres, Fisher et Jackson, 1831.

Grell, Chantal. Herculanum et Pompéi dans les récits de voyageurs français du XVIII ${ }^{e}$ siècle. Naples, Publication du Centre Jean Bérard, 1982. http://books.openedition.org/pcjb/233.

Grosley, Pierre Jean. Nouveaux mémoires, ou Observations sur l'Italie et les Italiens, par deux gentilshommes suédois. 3 vols. Londres : Jean Nourse, 1764.

Grosse, Christian. «Des « rites de passage » avant van Gennep : les cérémonies funéraires dans les traités antiquaires et « ethnographiques » de la première modernité (xvi ${ }^{\mathrm{e}}$-xviii ${ }^{\mathrm{e}}$ siècle) », Anabases, vol. 23, 2016, pp. 99-114. http://journals.openedition.org/anabases/5599.

Halpern, Jean-Claude. « Les Avatars de la barbarie sur les côtes du Golfe de Guinée, dans la première moitié du $18 \mathrm{e}$ siècle », Dix-huitième siècle, vol. 44, no. 1, 2012, pp. 147-164.

Hartog, François. Le Miroir d'Hérodote [1980], Paris, Gallimard, coll. Folio, 2001.

Iselin, Regula. "Reading Pictures: On the Value of the Copperplates in the 'Beschryvinghe' of Pieter De Marees (1602) as Source Material for Ethnohistorical Research." History in Africa, vol. 21, 1994, pp. 147-170. JSTOR, www.jstor.org/stable/3171884.

Labat, Jean-Baptiste. Voyages du Père Labat en Espagne et en Italie. 8 volumes. 
Paris: Delespine, 1730.

Law, Robin. "Human Sacrifice in Pre-Colonial West Africa." African Affairs, vol. 84, no. 334, 1985, pp. 53-87. JSTOR, www.jstor.org/stable/722523.

Le Huenen, Roland. «Qu'est-ce qu'un récit de voyage ? », Littérales $n^{\circ} 7$ : Les Modèles du récit de voyage, Nanterre, Université Paris X, 1990, p.11-27.

Letourneur, Pierre. Voyage à Ermenonville. Euvres complètes de J.-J. Rousseau, S.n (Poinçot), S.1. (Paris), 1788, vol.1, pp.59-176.

Meeres, Pieter (de). Verat et Historicam Descriptionem Auriferi Regni Guinea. Edité par Theodore de Bry dans Indiae Orientalis VI. Francfort, 1604.

---. Description et recit historial du riche royaume d'or de Gunea, aultrement nommé, la coste d'or de Mina. Amsterdamme [sic], Cornille Claesson, 1605.

---. A Description and historicall declaration of the Golden Kingdome of Guinea. Edité par Samuel Purchas dans Purchas His Pilgrimes. Edited in Five books. Londres, William Stansby, 1625, II, 926-970.

Milet de Mureau (de), Louis Marie. Voyage de La Pérouse autour du monde. 4 vols. Paris, Imprimerie de la République, 1797.

Misson, Maximilien. Nouveau voyage d'Italie fait en l'année 1688. 2 vols. La Haye, Henry van Bulderen, 1691.

Ouellet, Réal. « Sexualité et mariage dans les relations de voyages réels et imaginaires au début du XVIIIe siècle. " Sexualité, mariage et famille au XVIIIe siècle, édité par Olga B. Cragg et Rosena Davison, Presses de l'université Laval, 1998, pp. 27-51.

Pasquali, Adrien. Le Tour des Horizons. Critique et récits de voyage. Paris : Klincksieck: 1994.

Prévost, Antoine François. Histoire générale des voyages. 15 volumes. Paris: Didot, 1746-1759.

Rickman, John. Journal of Captain Cook's last voyage to the Pacific Ocean on Discovery: performed in the years 1776, 1777, 1778, 1779, illustrated with cuts, and a chart, shewing the tracts of the ships employed in this expedition. Londres, J. Newberry, 1781.

---. Troisième voyage de Cook ou Journal d'une expédition faite dans la mer pacifique du sud et du nord en 1776, 1777, 1778, 1779 et 1780. Paris, Poinçot et Belin, 1783.

Ridehalgh, Anne. "Rousseau as God: The Ermenonville Pilgrimages in the Revolution." Studies on Voltaire and the Eighteenth Century, vol. 278, 1990, pp. 287-308.

---. « Preromantic attitudes and the birth of a legend: French pilgrimage to Ermenonville », SVEC, CCXV, 1982 , pp. 231-252.

Stagl, Justin. A History of Curiosity: The Theory of Travel 1550-1800. Chur, Harwood Academic Publishers, 1995.

Trousson, Raymond. Jean-Jacques Rousseau jugé par ses contemporains. Du Discours sur les sciences et les arts aux Confessions. Paris, Honoré Champion, 2000.

Vijayan, Devika. « Les anecdotes du suttisme - image d'une altérité féminine 
ambiguë », Convergences francophones, vol. 3., no. 1, pp.10-21.

Vovelle, Michel. Mourir autrefois. [1974] Paris, Gallimard. Coll. Folio, 1994.

---. « Sur la mort. » Idéologies et mentalités. [1982] Paris, Gallimard. Coll. Folio, 1992, p.109-127.

---. « Les Attitudes devant la mort, front actuel de l'histoire des mentalités. » Archives de sciences sociales des religions, $\mathrm{n}^{\circ} 39,1975$, pp. 17-29.

Williams, Glyn. The Death of Captain Cook: A Hero Made and Unmade. Cambridge, Harvard University Press, 2008. 